\title{
Interferometric observations of the Mira star $o$ Ceti with the VLTI/VINCI instrument in the near-infrared ${ }^{\star}, \star \star$
}

\author{
H. C. Woodruff ${ }^{1}$, M. Eberhardt ${ }^{1}$, T. Driebe ${ }^{1}$, K.-H. Hofmann ${ }^{1}$, K. Ohnaka ${ }^{1}$, A. Richichi ${ }^{2}$, D. Schertl ${ }^{1}$ \\ M. Schöller ${ }^{3}$, M. Scholz ${ }^{4,5}$, G. Weigelt ${ }^{1}$, M. Wittkowski ${ }^{2}$, and P. R. Wood ${ }^{6}$ \\ 1 Max-Planck-Institut für Radioastronomie, Auf dem Hügel 69, 53121 Bonn, Germany \\ 2 European Southern Observatory, Karl-Schwarzschildt-Str. 2, 85748 Garching, Germany \\ 3 European Southern Observatory, Casilla 19001, Santiago 19, Chile \\ ${ }^{4}$ Institut für Theoretische Astrophysik, Universität Heidelberg, Tiergartenstr. 15, 69121 Heidelberg, Germany \\ 5 Institute of Astronomy, School of Physics, University of Sydney, NSW 2006, Australia \\ ${ }^{6}$ Research School of Astronomy and Astrophysics, Australian National University, Cotter Road, \\ Weston Creek ACT 2611, Australia
}

Received 9 December 2003 / Accepted 1 April 2004

\begin{abstract}
We present $K$-band commissioning observations of the Mira star prototype $o$ Cet obtained at the ESO Very Large Telescope Interferometer (VLTI) with the VINCI instrument and two siderostats. The observations were carried out between 2001 October and December, in 2002 January and December, and in 2003 January. Rosseland angular radii are derived from the measured visibilities by fitting theoretical visibility functions obtained from center-to-limb intensity variations (CLVs) of Mira star models (Bessell et al. 1996; Hofmann et al. 1998; Tej et al. 2003b). Using the derived Rosseland angular radii and the SEDs reconstructed from available photometric and spectrophotometric data, we find effective temperatures ranging from $T_{\text {eff }}=3192 \pm 200 \mathrm{~K}$ at phase $\Phi=0.13$ to $2918 \pm 183 \mathrm{~K}$ at $\Phi=0.26$. Comparison of these Rosseland radii, effective temperatures, and the shape of the observed visibility functions with model predictions suggests that $o$ Cet is a fundamental mode pulsator. Furthermore, we investigated the variation of visibility function and diameter with phase. The Rosseland angular diameter of $o$ Cet increased from $28.9 \pm 0.3$ mas (corresponding to a Rosseland radius of $332 \pm 38 R_{\odot}$ for a distance of $D=107 \pm 12 \mathrm{pc}$ ) at $\Phi=0.13$ to $34.9 \pm 0.4$ mas $\left(402 \pm 46 R_{\odot}\right)$ at $\Phi=0.4$. The error of the Rosseland linear radius almost entirely results from the error of the parallax, since the error of the angular diameter is only approximately $1 \%$.
\end{abstract}

Key words. instrumentation: interferometers - techniques: interferometric - stars: late-type - stars: AGB and post-AGB stars: fundamental parameters - stars: individual: Mira

\section{Introduction}

Mira stars are long-period variables (LPVs) which evolve along the asymptotic giant branch (AGB), and are characterized by stellar pulsation with amplitudes as large as $\Delta V \sim 9$ and welldefined pulsation periods (80-1000 days). In recent years, the comparison of theoretical pulsation models with MACHO observations of LPVs in the LMC, in particular the reproduction of period ratios in multimode pulsators, has shown that Miras are fundamental-mode pulsators (Wood et al. 1999). However, radius measurements of Mira variables when compared to theoretical pulsation calculations have generally yielded the large

Send offprint requests to: $\mathrm{H}$. C. Woodruff, e-mail: woodruff@mpifr-bonn.mpg.de

* Based on observations collected at the European Southern Observatory, Paranal, Chile (public commissioning data).

$\star \star$ Based on data collected at the Special Astrophysical Observatory (SAO), Russia. values expected for first overtone pulsators (e.g., Feast 1996; van Belle et al. 1996). There is clearly a problem with the interpretation of radius measurements that needs examination.

High-resolution interferometric studies of Mira stars allow the determination of the size of the stellar disk, its centerto-limb intensity variation, surface inhomogeneities, and the dependence of diameter on wavelength and variability phase (see, e.g., Pease 1931; Bonneau \& Labeyrie 1973; Labeyrie et al. 1977; Bonneau et al. 1982; Karovska et al. 1991; Haniff et al. 1992; Quirrenbach et al. 1992; Wilson et al. 1992; Tuthill et al. 1994; Danchi et al. 1994; Haniff et al. 1995; Weigelt et al. 1996; van Belle et al. 1996; Burns et al. 1998; Perrin et al. 1999; Hofmann et al. 2000b; Weigelt et al. 2000; Weiner et al. 2000; Thompson et al. 2002; Meisner 2003). The results of such interferometric observations can be compared with predictions from theoretical models of stellar pulsation and the atmosphere of Mira stars (e.g., Watanabe \& Kodaira 1979; Scholz 1985; Bessell et al. 1989, 1996 = BSW; 
Table 1. Summary of VINCI commissioning observations of $o$ Cet: date, Julian Date JD, cycle and visual phase $\Phi$ (see Fig. 1), number of visibility data points $N$, projected baseline length $B_{\mathrm{p}}$, and baseline projection angle PA.

\begin{tabular}{lccccc}
\hline \hline Date & JD & Cycle $+\Phi$ & $N$ & $B_{\mathrm{p}}[\mathrm{m}]$ & PA [ $\left.{ }^{\circ}\right]$ \\
\hline 2001 Oct. 22 & 2452205 & 0.13 & 13 & $11.5-16.0$ & $62-73$ \\
2001 Oct. 23 & 2452206 & 0.13 & 12 & $11.5-16.0$ & $63-73$ \\
2001 Nov. 09 & 2452223 & 0.18 & 6 & $14.5-16.0$ & $71-73$ \\
2001 Nov. 15 & 2452229 & 0.18 & 3 & $15.5-16.0$ & $72-73$ \\
2001 Nov. 17 & 2452231 & 0.18 & 3 & $14.5-15.0$ & 70 \\
2001 Dec. 06 & 2452250 & 0.26 & 6 & $15.0-16.0$ & $72-73$ \\
2002 Jan. 20 & 2452295 & 0.40 & 2 & $14.0-14.5$ & 72 \\
2002 Dec. 20 & 2452629 & 1.40 & 2 & $7.5-8.0$ & $73-74$ \\
2003 Jan. 09 & 2452649 & 1.47 & 1 & 8.0 & 73 \\
\hline
\end{tabular}

Hofmann et al. $1998=$ HSW; Tej et al. 2003b = TLSW; Ireland et al. $2004=$ ISW). Confrontation of detailed theoretical models with high-resolution observations is crucial for improving our understanding of the physical properties of Mira stars (e.g., Hofmann et al. 2000a, 2001; Weigelt et al. 2003; Scholz 2003).

In this paper we present ESO VLTI/VINCI visibility measurements of $o$ Cet and compare the measured visibility shape and the phase dependence of the visibility with model predictions. $o$ Cet, the prototype of oxygen-rich Mira stars, is a very suitable object for these studies, since VINCI observations exist for different phases and baselines, its distance is known (revised HIPPARCOS distance $107.06 \pm 12.26$ pc, Knapp et al. 2003), and a large amount of spectroscopic and photometric data is available for different phases.

\section{Observations and data reduction}

\subsection{Observations}

A total of 48 visibility measurements of $o$ Cet were carried out with VINCI (Kervella et al. 2003) at the VLTI in the commissioning period between 2001 October and 2003 January. All this data has been publicly released. Projected baselines ranging from 7.5 to $16.0 \mathrm{~m}$ were employed. VINCI is a fiberoptics beam combiner instrument based on the concept of the FLUOR instrument (Coudé du Foresto \& Ridgway 1992; Mariotti et al. 1996). With the single-mode fibers to spatially filter the wavefronts perturbed by atmospheric turbulence, this beam combiner provides accurate visibility measurements in spite of time-variable atmosphere conditions.

Table 1 gives an overview of the VINCI observations. Figure 1 shows the visual light curve of $o$ Cet together with the dates at which the VINCI observations were carried out (Mattei 2003). Figure 2 shows the visibilities of $o$ Cet vs. spatial frequency measured at six different phases. The comparison between the observations and the different Mira star model series will be discussed in Sect. 3 .

In addition to the VINCI observations, we recorded speckle interferograms of $o$ Cet on 2003 October 7 with the SAO $6 \mathrm{~m}$ telescope in Russia. The speckle camera used for the

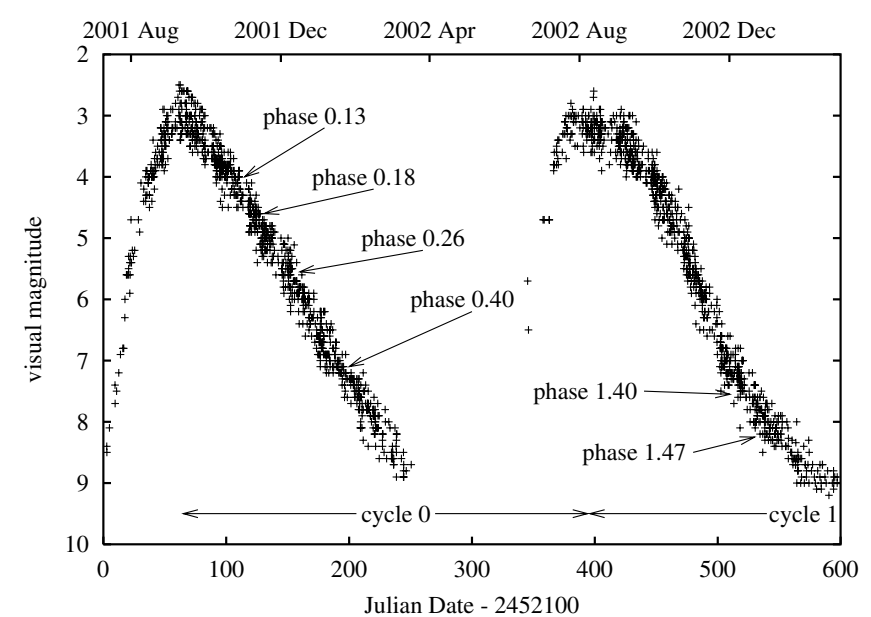

Fig. 1. Visual light curve for $o$ Cet (Mattei 2003) between JD 2452100 and 2452700 . The VINCI visibilities were measured at the following phases: $\Phi=0.13$ (2001 Oct. 22/23), $\Phi=0.18$ (2001 Nov. 09/15/17), $\Phi=0.26$ (2001 Dec. 06), $\Phi=0.40$ (2002 Jan. 20), $\Phi=1.40$ (2002 Dec. 20), and $\Phi=1.47$ (2003 Jan. 09). Phases larger than 1 indicate that the observations belong to the next pulsation cycle.

observations was equipped with a Rockwell HAWAII array. The field of view of the recorded speckle interferograms was $11^{\prime \prime} .02$. A filter with a central wavelength of $2086 \mathrm{~nm}$ and a width of $20 \mathrm{~nm}$ was used. The exposure time per frame was $10 \mathrm{~ms}$. The complete data set consists of 100 speckle interferograms of $o$ Cet and 360 of an unresolved reference star (HD 14652). The seeing (FWHM) was $\sim 3$ ". 4 . The visibilities were obtained using the speckle interferometry method (Labeyrie 1970).

\subsection{Data reduction}

Processing of the raw VINCI data for estimating the coherence factors $\mu$ (i.e. the fringe contrast or uncalibrated visibility) was carried out with the VINCI data reduction software provided by the European Southern Observatory ${ }^{1}$ (version 2.0.6), based on wavelets transforms to derive the power spectral density. The processing of a series of OPD scans yields the uncalibrated squared visibility $\mu^{2}$ for each individual scan, and the averaged value $\left\langle\mu^{2}\right\rangle$ is used to derive the calibrated visibility (Coudé du Foresto et al. 1997; Perrin 2003). The squared transfer functions $T^{2}$, which account for instrumental and atmospheric effects, were derived from measurements of calibrator stars and their known angular diameters. The expected calibrator visibility $V_{\text {cal }}$ was calculated from uniform disk (UD) angular diameters of the calibrators given in Richichi \& Percheron (2002), and the transfer function was evaluated as

$T^{2}=\frac{\mu_{\mathrm{cal}}^{2}}{V_{\mathrm{cal}}^{2}}$,

where $\mu_{\text {cal }}$ is the measured uncalibrated visibility of the calibrator. The calibrators of the nights on which $o$ Cet was measured are listed in Table 2. According to Eq. (1), the errors of the

\footnotetext{
1 www. eso.org/ pballest/vinci
} 

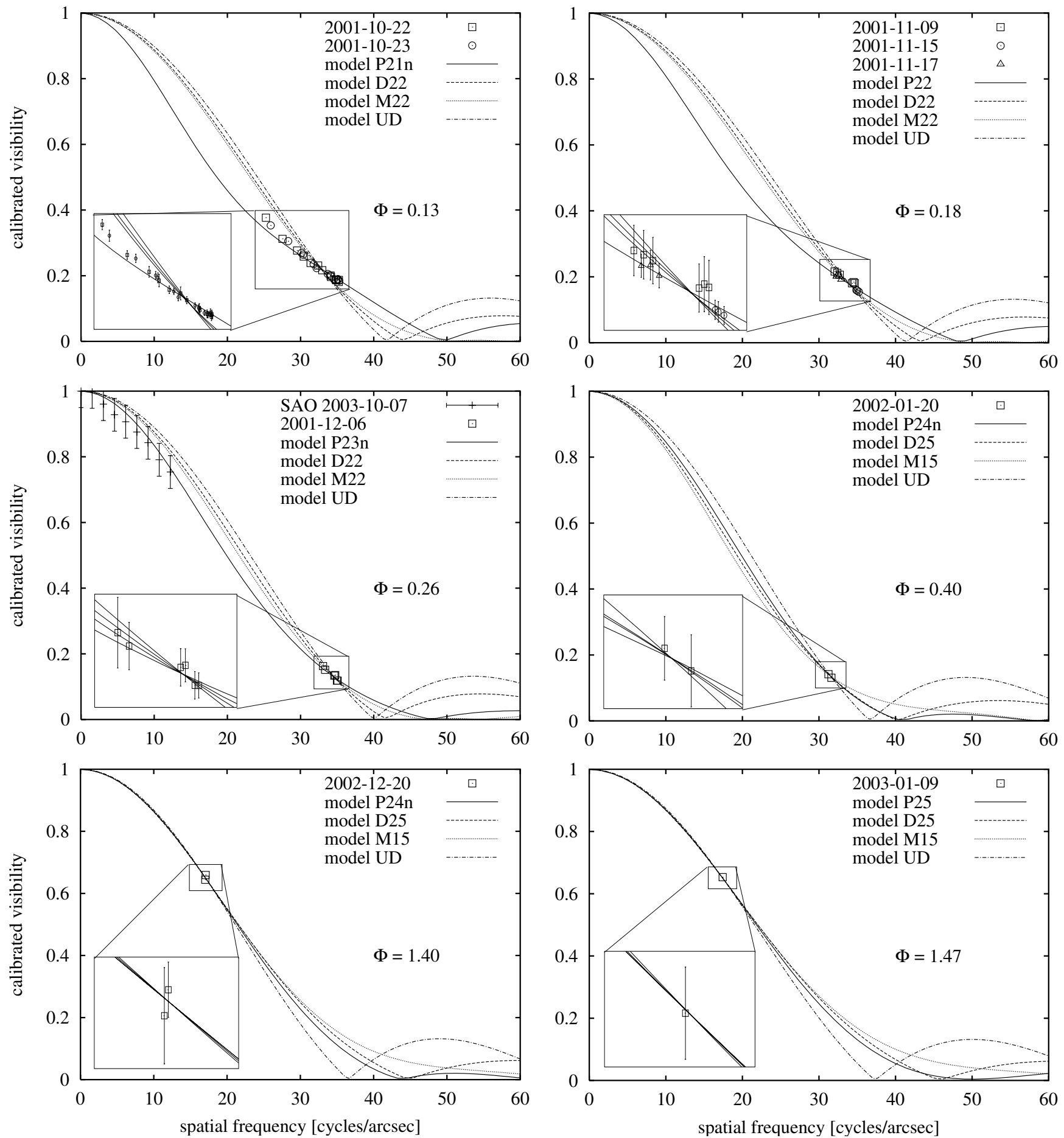

Fig. 2. Visibilities of $o$ Cet vs. spatial frequency measured at phase 0.13 (top left, observation dates 2001 Oct. 22/23), at phase 0.18 (top right, observation dates 2001 Nov. 09/15/17), at phase 0.26 (middle left, observation date 2001 Dec. 06) together with SAO speckle interferometry data measured at the same phase but at a different cycle (observation date 2003 Oct. 07; data not used for the model visibility fit), at phase 0.40 (middle right, observation date 2002 Jan. 20), at phase 1.40 (bottom left, observation date 2002 Dec. 20), and at phase 1.47 (bottom right, observation date 2003 Jan. 09). The insets show an enlargement of the relevant spatial frequency range. Here, the error bars are included. The fits with different Mira star models are discussed in Sect. 3. Solid lines represent the P models from TLSW and ISW, dashed lines the D models from BSW, dotted lines the M models from HSW, and the dash-dotted lines represent the simple uniform-disk model CLVs. Note that from our SAO observations there is no indication for a visibility contribution from a circumstellar dust shell at low spatial frequencies.

individual transfer function values were calculated from the errors of the raw visibilities and the errors of the uniform-disk visibilities of the calibrators which arise from the uncertainties of the corresponding uniform-disk diameters. For most of the individual transfer function values, the error contributions of the raw visibility and the uniform-disk visibility of the calibrator are of the same order, resulting in total errors of typically $\$ 5 \%$ (see Fig. 3 ). Only in a few 
Table 2. List of calibrators used (see Richichi \& Percheron 2002) and their UD angular diameters $d_{\mathrm{UD}}^{\mathrm{a}}$.

\begin{tabular}{|c|c|c|c|}
\hline Calibrators & $d_{\mathrm{UD}}^{\mathrm{a}}[\mathrm{mas}]$ & Nights & Sp. type \\
\hline $30 \mathrm{Psc}$ & $7.20 \pm 0.70$ & 2001 Oct. 22 & M3III \\
\hline$\tau \mathrm{Aqr}$ & $4.97 \pm 0.05$ & 2001 Oct. 22,23 & K5III \\
\hline$\gamma$ Eri & $8.51 \pm 0.09$ & $\begin{array}{l}2001 \text { Oct. } 22,23, \\
2001 \text { Nov. } 09,15 \text {, } \\
2001 \text { Dec. } 06, \\
2002 \text { Dec. } 20\end{array}$ & M1IIIb \\
\hline$\alpha$ Cet & $11.52 \pm 0.29$ & $\begin{array}{l}2001 \text { Oct. } 22 \text {, } \\
2002 \text { Dec. } 20\end{array}$ & M1.5IIIa \\
\hline$\chi \mathrm{Aqr}$ & $6.70 \pm 0.15$ & 2001 Oct. 23 & M3III \\
\hline$\tau$ Pup & $4.49 \pm 0.07$ & $\begin{array}{l}2001 \text { Oct. 23, } \\
2002 \text { Jan. } 20, \\
2002 \text { Dec. } 20 \text {, } \\
2003 \text { Jan. } 09\end{array}$ & K1III \\
\hline$\pi$ Eri & $4.80 \pm 0.50$ & 2001 Nov. $09,15,17$ & M1III \\
\hline Sirius & $5.60 \pm 0.15$ & $\begin{array}{l}2001 \text { Nov. } 09,15,17 \text {, } \\
2002 \text { Jan. } 20, \\
2002 \text { Dec. } 20\end{array}$ & $\mathrm{~A} 1 \mathrm{~V}$ \\
\hline$\beta$ Cet & $5.18 \pm 0.06$ & $\begin{array}{l}2001 \text { Oct. } 22,23, \\
2001 \text { Nov. } 15,17 \text {, } \\
2001 \text { Dec. } 06, \\
2002 \text { Dec. } 20\end{array}$ & K0III \\
\hline$\delta \mathrm{CMa}$ & $3.29 \pm 0.46$ & 2001 Nov. 15 & F8Iab \\
\hline HD 12274 & $5.30 \pm 0.50$ & 2002 Jan. 20 & K5/M0III \\
\hline$\theta \mathrm{CMa}$ & $4.13 \pm 0.40$ & $\begin{array}{l}2002 \text { Jan. } 20 \\
2002 \text { Dec. } 20\end{array}$ & K4III \\
\hline$\alpha$ Hya & $9.44 \pm 0.90$ & $\begin{array}{l}2002 \text { Jan. } 20 \text {, } \\
2003 \text { Jan. } 09\end{array}$ & K3II-III \\
\hline$\pi$ Leo & $4.78 \pm 0.26$ & 2002 Jan. 20 & M2III \\
\hline$\eta$ Cet & $3.35 \pm 0.04$ & 2002 Dec. 20 & K1.5III \\
\hline$\epsilon$ Lep & $5.90 \pm 0.06$ & $\begin{array}{l}2001 \text { Oct. } 22,23 \\
2002 \text { Dec. } 20 \text {, } \\
2003 \text { Jan. } 09\end{array}$ & K4III \\
\hline$\zeta$ Hya & $3.10 \pm 0.20$ & 2003 Jan. 09 & G9II-III \\
\hline$\iota$ Hya & $3.41 \pm 0.05$ & 2003 Jan. 09 & $\mathrm{~K} 2.5 \mathrm{III}$ \\
\hline$\mu$ Нyа & $4.69 \pm 0.50$ & 2003 Jan. 09 & K4III \\
\hline$\beta \mathrm{Cnc}$ & $4.88 \pm 0.03$ & 2003 Jan. 09 & K4III \\
\hline
\end{tabular}

cases the error contribution of either the raw visibility or the uniform-disk visibility of the calibrator is significantly larger, resulting in much larger errors of the transfer function values.

In order to obtain the squared transfer function for the time of the observation of the object, the measured values of the squared transfer function $T^{2}$ of each night were plotted against the time of observation and fitted by a straight line. Since the transfer function proved to be a slowly varying function which was rapidly sampled, as Fig. 3 shows, we did not apply higher order polynomial fits. The error of the time-dependent transfer function is given by the error of the linear interpolation. To estimate the error introduced by our method of determining the transfer function at the time of the observation of $o$ Cet, we also applied constant fits to the three nights where the variation of the transfer values was largest (2001 Dec. 06, 2002 Jan. 20, and 2003 Jan. 09). The difference obtained for the $o$ Cet calibrated visibilities using the linear and the constant transfer function fit procedures is $1.5 \%$ at most, leading to a final error of 0.1 mas for the Rosseland diameter of $o$ Ceti.

Since VINCI uses a $K$ broad-band filter, model visibilities calculated with VINCI's filter response function would be appropriate for the comparison of models and measurements. However, those polychromatic VINCI-filter models cannot be calculated since monochromatic Mira star CLV models are not readily available. Therefore, instead of using VINCI-filter model CLVs, we adopted the following approximative scheme: In our visibility derivations we assumed the same effective wavelength $\lambda_{\mathrm{eff}}=2.2 \mu \mathrm{m}$ for all calibrators and science objects. This effective wavelength results from the assumption of a simple rectangular response function for the $K$-band filter used (central wavelength $2.2 \mu \mathrm{m}$ and bandwidth $0.4 \mu \mathrm{m}$ ) and a constant spectrum of the calibrators/science objects within the $K$ band. This simplification introduces only a minor error in our calculations: the effective wavelength $\lambda_{\text {eff }}$ will be different from $2.2 \mu \mathrm{m}$ since the filter response function is not rectangular in shape (see, e.g., Wittkowski et al. 2004), and the calibrator stars have diverse spectra. Our calculations using the calibrator star with the earliest spectral type (Sirius) lead to an error of the calibrated object visibility of $0.23 \%$ at $16 \mathrm{~m}$ baseline (the largest baseline for the observations discussed in this paper).

The final errors (Fig. 2) of the calibrated visibilities were derived from simple Gaussian error propagation of the error of the fits to the measured transfer function values and the error of the uncalibrated visibility together with the additional effective wavelength error.

\section{Comparison of the observations with Mira star models}

\subsection{Mira star models}

All Mira star models used in this paper are from BSW (D and E series), HSW (P, M, and O series), TLSW (P series), and from ISW (P series). They were developed as possible representations of $o$ Cet and hence have periods $P$ close to 332 days. These models differ in pulsation mode, stellar mass $M$, parent star radius $R_{\mathrm{p}}$ (i.e., radius of the initial non-pulsating model), and luminosity $L$. The parent-star luminosities of the model series had been chosen based on considerations of the period- luminosity-relation for the LMC and a theoretical metallicity correction (BSW, HSW). Solar abundances were assumed for all models. The five model series represent stars pulsating in the fundamental mode (f; D, P, and M) or in the first overtone mode $(o ; \mathrm{E}$ and $\mathrm{O})$. Table 3 lists the properties of the non-pulsating parent stars of these Mira models, and Table 4 shows the Rosseland radius and effective temperature 

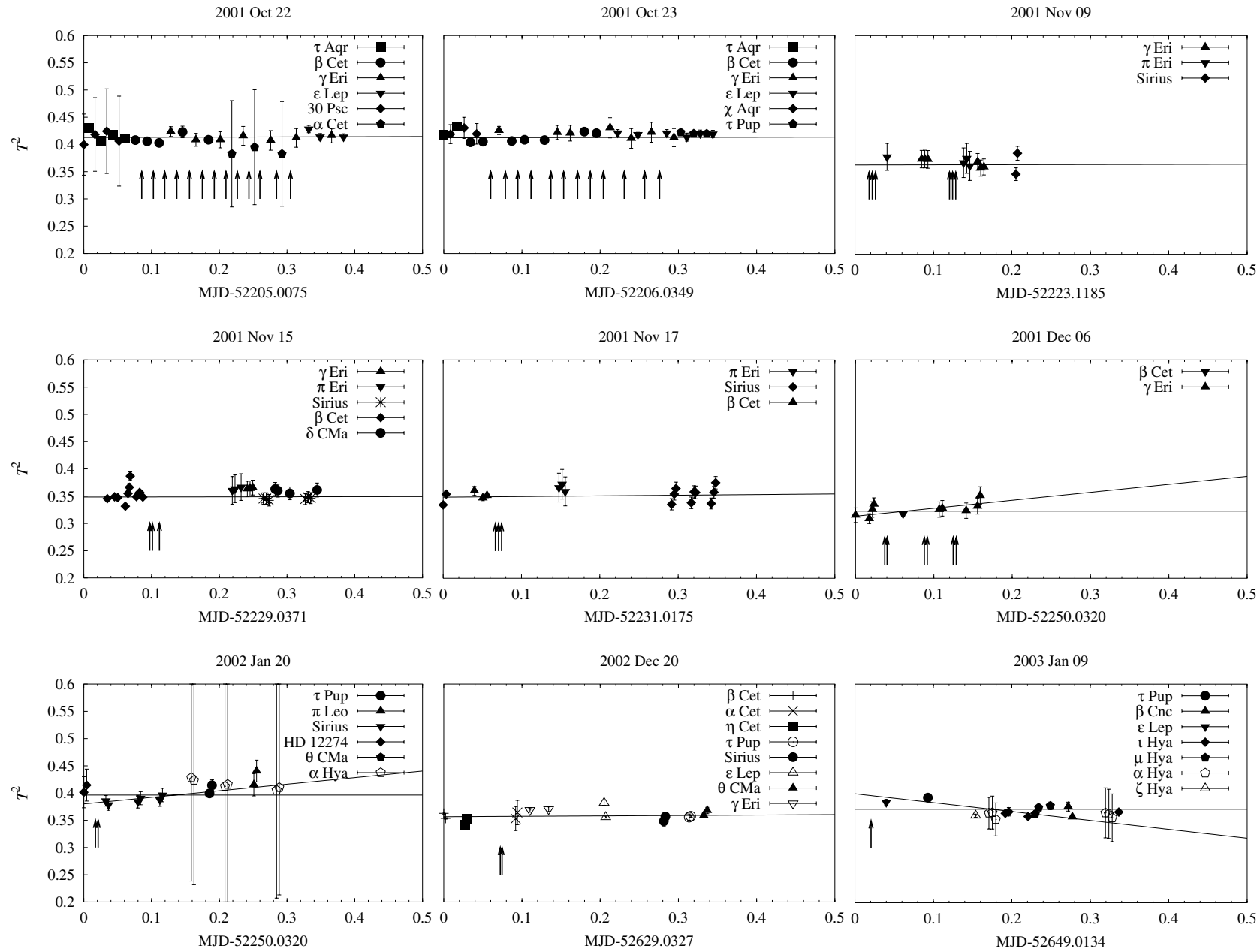

Fig. 3. Linearly fitted squared transfer functions (see text) as a function of time (MJD = Modified Julian Date) obtained from calibrator stars measured (see Table 2). For comparison, in the nights of 2001 Dec. 06, 2002 Jan. 20 and 2003 Jan. 09 we included a constant fit of the squared transfer functions. The arrows mark the observation times of $o$ Cet.

Table 3. Properties of Mira model series from BSW, HSW, ISW, and TLSW (see text). $L$ is the luminosity, $M$ the stellar mass, and $P$ the pulsation period in days. $R_{\mathrm{p}}$ is the Rosseland radius of the initial non-pulsating "parent" model of the Mira variable, and $\alpha$ is the mixing-length parameter of convection (= the mixing-length in units of the local pressure scale height, see Böhm-Vitense 1958).

\begin{tabular}{cccccccc}
\hline \hline Series & Mode & $P(\mathrm{~d})$ & $M / M_{\odot}$ & $L / L_{\odot}$ & $R_{\mathrm{p}} / R_{\odot}$ & $T_{\text {eff }} / \mathrm{K}$ & $\alpha$ \\
\hline D & $\mathrm{f}$ & 330 & 1.0 & 3470 & 236 & 2900 & 1.76 \\
E & o & 328 & 1.0 & 6310 & 366 & 2700 & 1.26 \\
P & f & 332 & 1.0 & 3470 & 241 & 2860 & 2.06 \\
M & f & 332 & 1.2 & 3470 & 260 & 2750 & 1.73 \\
O & o & 320 & 2.0 & 5830 & 503 & 2250 & 0.93 \\
\hline
\end{tabular}

associated with all P-series models. All models are named after the cycle (see Fig. 1 for the definition of cycle) and phase they represent. For example, P28 denotes the P model calculated near visual phase 0.8 in the second cycle. The suffix " $n$ ", (e.g., P11n), denotes the new models from the recent fine phase grid of ISW.

In this paper we use the conventional photospheric stellar Rosseland radius $R_{\text {Ross }}$ given by the distance from the star's centre at which the Rosseland optical depth equals unity. This Rosseland radius is no observable quantity (cf. the discussion in Baschek et al. 1991; Scholz 2003). Usually, it is close to the near-infrared radius measured in near-continuum bandpasses except at near-minimum phases with strong molecular bandpass contamination (HSW, Jacob \& Scholz 2002; Scholz 2003). Therefore, for better comparison Table 4 also gives the $R_{1.04}$ radius that is defined by the position of the $\tau=1$ layer in the $1.04 \mu \mathrm{m}$ continuum window.

In order to derive the model visibilites, we averaged the $K$-band model CLV over all monochromatic CLVs between $2.0 \mu \mathrm{m}$ and $2.4 \mu \mathrm{m}$. An optimal comparison with 
Table 4. The variability phase $\Phi$, Rosseland radius $R_{\text {Ross }}$ in units of the parent star radius $R_{\mathrm{p}}, K$-band radius $R_{K}$ in units of the Rosseland radius, radius $R_{1.04}$, which is defined by the position of the $\tau=1$ layer in the $1.04 \mu \mathrm{m}$ continuum window in units of $R_{\mathrm{p}}$, the effective temperature $T_{\text {eff }}(R)$, and the luminosity $L$ for all P-series models from HSW and TLSW. Models denoted by "n" are from ISW with finer phase spacing (see text). Phases in the $1+0.0$ to $3+0.0$ range were re-assigned as described in ISW.

\begin{tabular}{llllllc}
\hline \hline Model & Cycle+ $\Phi$ & $R_{\text {Ross }} / R_{\mathrm{p}}$ & $R_{K} / R_{\text {Ross }}$ & $R_{1.04} / R_{\mathrm{p}}$ & $\begin{array}{c}T_{\text {eff }}(R) \\
{[\mathrm{K}]}\end{array}$ & $\begin{array}{c}L \\
{\left[L_{\odot}\right]}\end{array}$ \\
\hline P05 & $0+0.5$ & 1.20 & 0.65 & 0.90 & 2160 & 1650 \\
P08 & $0+0.8$ & 0.74 & 0.97 & 0.74 & 3500 & 4260 \\
P10 & $1+0.00$ & 1.03 & 0.94 & 1.04 & 3130 & 5300 \\
P11n & $1+0.10$ & 1.17 & 0.88 & 1.19 & 2990 & 5650 \\
P12 & $1+0.23$ & 1.38 & 0.78 & 1.30 & 2610 & 4540 \\
P13n & $1+0.30$ & 1.53 & 0.69 & 1.26 & 2310 & 3450 \\
P14n & $1+0.40$ & 1.73 & 0.58 & 1.19 & 2080 & 2920 \\
P15n & $1+0.50$ & 1.88 & 0.49 & 0.84 & 1800 & 1600 \\
P15 & $1+0.60$ & 1.49 & 0.47 & 0.85 & 1930 & 1910 \\
P18 & $1+0.87$ & 0.77 & 0.94 & 0.77 & 3520 & 4770 \\
P20 & $1+0.99$ & 1.04 & 0.92 & 1.04 & 3060 & 4960 \\
P21n & $2+0.11$ & 1.23 & 0.87 & 1.21 & 2790 & 4750 \\
P22 & $2+0.18$ & 1.32 & 0.82 & 1.26 & 2640 & 4400 \\
P23n & $2+0.30$ & 1.36 & 0.78 & 1.24 & 2470 & 3570 \\
P24n & $2+0.40$ & 1.38 & 0.72 & 1.16 & 2210 & 2380 \\
P25 & $2+0.53$ & 1.17 & 0.66 & 0.91 & 2200 & 1680 \\
P28 & $2+0.83$ & 0.79 & 0.97 & 0.79 & 3550 & 5200 \\
P30 & $2+0.98$ & 1.13 & 0.89 & 1.14 & 3060 & 5840 \\
P35 & $3+0.5$ & 1.13 & 0.63 & 0.81 & 2270 & 1760 \\
P38 & $3+0.8$ & 0.78 & 0.96 & 0.78 & 3570 & 5110 \\
P40 & $4+0.0$ & 1.17 & 0.90 & 1.16 & 2870 & 4820 \\
\hline & & & & & & \\
\hline
\end{tabular}

VINCI visibilities, however, would require a slightly different calculation of the polychromatic model visibility function: for each monochromatic model CLV within the $K$ band, first the squared visibility function has to be calculated, and then averaging over the entire wavelength range will deliver the correct squared visibility function. The square root of this average corresponds to the visibility curve measured with VINCI (see, e.g., Wittkowski et al. 2004). Comparison of the two methods for obtaining model visibility curves shows only small discrepancies in the relevant spatial frequency range and yields a systematic visibility difference of less than $0.5 \%$, which we included in the error budget of the calibrated visibility.

The Rosseland angular radii $R_{\text {Ross }}^{\mathrm{a}}$ of $o$ Cet were determined in the following way: first, we derived the $K$-band angular radius $R_{K}^{\mathrm{a}}$, which is defined as the FWHM of the model CLV. The $R_{K}^{\mathrm{a}}$ values (corresponding to different model-phase combinations) were determined by least-square fits between the measured Mira visibilities and the visibilities of the corresponding model CLVs. Then, the Rosseland angular radii $R_{\text {Ross }}^{\mathrm{a}}$ were derived from the obtained $K$-band angular radii and from the theoretical ratios $R_{\text {Ross }} / R_{K}$ provided by the models. For a more detailed description of the visibility fitting procedure we refer to HSW.

\subsection{Comparison of observed and model Rosseland linear radii}

Linear Rosseland radii of $o$ Cet were obtained using the derived angular radii together with Mira's revised HIPPARCOS parallax of $9.34 \pm 1.07$ mas (Knapp et al. 2003; ESA 1997).

Figure 4 shows the comparison between the derived Rosseland linear radii and those of the corresponding models which provided the CLVs. The $x$ axis represents different combinations of model series, phase, and cycles. Clearly, the E and $\mathrm{O}$ first-overtone series and the $\mathrm{D}$ fundamental-mode series predict radii that are systematically either too small (D series) or too large ( $\mathrm{O}$ and $\mathrm{E}$ series). The fundamental-mode $\mathrm{M}$ series shows good agreement for the phases $\Phi=0.13-0.26$ and 1.47, whereas the predicted radii are systematically too small at the phases 0.4 and 1.4 . The disagreement can be partially explained by the lack of $\mathrm{M}$ models for phases close to 0.4 , since we use near-minimum (M05 and M 15) models for the comparison. The absence of a fine phase grid also applies to the D and $\mathrm{O}$ model series, but agreement would probably not be improved with a finer phase grid, considering the large discrepancy between near-phase radii predictions and derived radii for $\Phi=0.18,0.26$, and 1.47. The P-series models from HSW, TLSW, and ISW predict, in most cases (i.e., 10 out of 14 cyclephase combinations), Rosseland linear radii which agree with the measured values within the error bars. This good agreement of the P models is independent of the phase grid spacing. The Rosseland linear radii predicted from the $\mathrm{M}$ models agree with the measured values (within the error bars) in 8 out of 12 cycle-phase combinations. This radius comparison favors the fundamental-mode pulsation $\mathrm{P}$ and $\mathrm{M}$ models as possible representations of Mira, whereas all first overtone pulsation model series can be ruled out as models that reliably predict the Rosseland radius of $o$ Cet.

\subsection{Determination of $T_{\text {eff }}$}

The effective temperature $T_{\text {eff }}$ of a star can be derived from its Rosseland angular diameter and its bolometric flux (cf. Scholz \& Takeda 1987; Baschek et al. 1991; Scholz 2003 for the issue of $T_{\text {eff }}$ definition). Using convenient units, the relation between $T_{\text {eff }}, F_{\text {bol }}$, and Rosseland angular diameter $d_{\text {Ross }}^{\text {a can be }}$ written as follows:

$T_{\text {eff }}=2341\left(\frac{F_{\text {bol }}}{10^{-11} \mathrm{~W} \mathrm{~m}^{-2}}\right)^{\frac{1}{4}}\left(\frac{d_{\text {Ross }}^{\mathrm{a}}}{\text { mas }}\right)^{-\frac{1}{2}} \mathrm{~K}$.

In order to estimate the bolometric flux, we used photometric measurements of $o$ Cet in the visual (Mattei 2003) and, when available, in the NIR in combination with the ISO spectrum ranging from $2.36 \mu \mathrm{m}$ to $45.38 \mu \mathrm{m}$. In the region between $0.7812 \mu \mathrm{m}$ and $1.0834 \mu \mathrm{m}$ spectrophotometric data were taken from measurements of Wing (2003). The 1.25 to $2.2 \mu \mathrm{m}$ data were taken from $J H K$ photometry by Yudin (2003). Since 

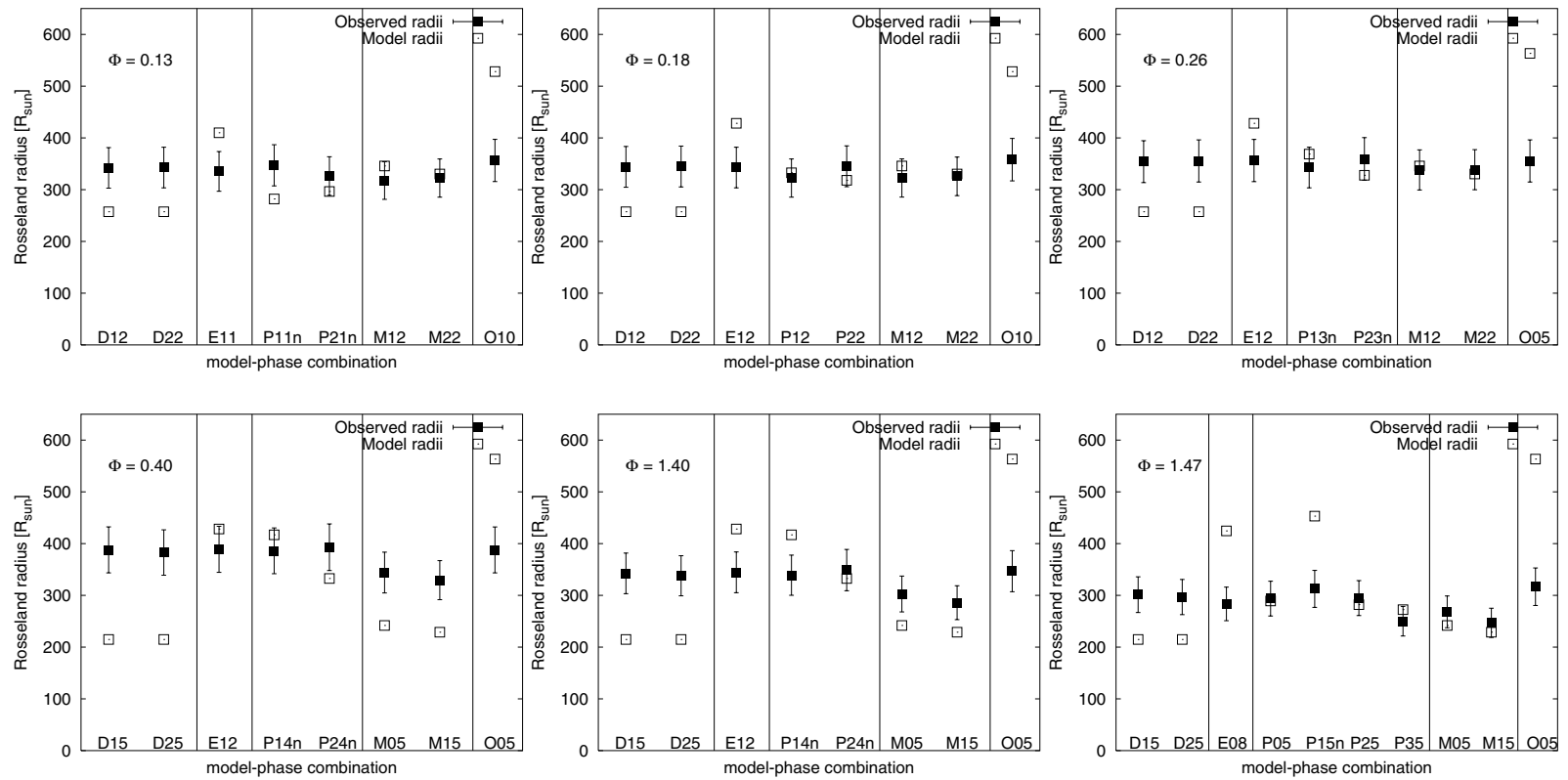

Fig. 4. Derived Rosseland linear radii for all epochs of observation and the Rosseland linear radii predicted by the nearest-phase models from all series. The Rosseland linear radii are given for the six measured phases $\Phi=0.13$ (top left), $\Phi=0.18$ (top right), $\Phi=0.26$ (middle left), $\Phi=0.40$ (middle right), $\Phi=1.40$ (bottom left), and $\Phi=1.47$ (bottom right). Open squares show the model values, while the filled squares with the error bars give the values derived from the observations. The large error bars of $10-15 \%$ are mainly due to the parallax error because the error contribution of the interferometric measurements is only approximately $1 \%$.

the ISO spectrum was taken at maximum light $(\Phi=0)$, we used light curves (Smith et al. 2002) obtained with the Diffuse Infrared Background Experiment (DIRBE, see Smith 2003) to estimate the variability of $o$ Cet in the ISO spectral range. DIRBE light curves are available in seven infrared bands between $1.25 \mu \mathrm{m}$ and $60 \mu \mathrm{m}$, giving us important complementary photometric data. Additional data used and the overall shape of the spectral energy distributions (SEDs) can be seen in Fig. 5.

The light curves in the ISO range show that flux variations in the mid- and far-infrared between maximum and minimum light is small but not negligible. Since most of the bolometric flux comes from the NIR, the error of $F_{\text {bol }}$ induced by flux density changes in the ISO range makes a contribution of only $\sim 5-10 \%$ to the total error. Because of the small interstellar extinction in the $V$ and $K$ bands $\left(A_{V}=0.09\right.$, Robertson \& Feast 1981, and $A_{K}=0.05$, Knapp et al. 2003) and the proximity of Mira, no correction for interstellar extinction was applied. Integration of the SED was made by simple trapezoid integration and yields $F_{\text {bol }}=2.63 \times 10^{-8} \mathrm{~W} \mathrm{~m}^{-2}$ at $\Phi=0.13, F_{\text {bol }}=2.41 \times 10^{-8} \mathrm{~W} \mathrm{~m}^{-2}$ at $\Phi=0.18$, and $F_{\text {bol }}=2.36 \times 10^{-8} \mathrm{~W} \mathrm{~m}^{-2}$ at $\Phi=0.26$. The total error of the bolometric flux was estimated to be $25 \%$. The error sources are mainly the cycle and phase uncertainties of the spectrophotometric and photometric observations. Since spectrophotometric and photometric data were not sufficiently available for reconstructing SEDs at phases later than 0.3, we derived $T_{\text {eff }}$ from the Rosseland angular diameter $d_{\text {Ross }}^{\mathrm{a}}$ and the SED according to Eq. (2) only for the phases $\Phi=0.13,0.18$, and 0.26 . The error in $T_{\text {eff }}$ is given by

$\frac{\Delta T_{\text {eff }}}{T_{\text {eff }}}=\frac{1}{2}\left[\left(\frac{\Delta d_{\text {Ross }}^{\mathrm{a}}}{d_{\text {Ross }}^{\mathrm{a}}}\right)^{2}+\frac{1}{4}\left(\frac{\Delta F_{\text {bol }}}{F_{\text {bol }}}\right)^{2}\right]^{\frac{1}{2}}$ and is $6 \%$ when errors of approximately $25 \%$ and $1 \%$ are assumed for $F_{\text {bol }}$ and $d_{\text {Ross }}^{\mathrm{a}}$, respectively.

Figure 6 presents $T_{\text {eff }}$ values derived at phases close to the VINCI observations from the bolometric flux and the Rosseland angular diameters. The $T_{\text {eff }}$ values of the $\mathrm{D}$ model series are very close (within the error bars) to those derived from the observations at all phases. Although the P11n model shows agreement between observation and theory for phase 0.13 , all other cycle-phase combination of the P-model series as well as all other model series yield large differences between theoretical and measured effective temperature values.

We also determined the luminosity range of $o$ Cet for the observed phases from its bolometric flux and HIPPARCOS parallax (9.34 \pm 1.07 mas, Knapp et al. 2003). We obtained $9360 \pm$ $3140 L_{\odot}$ for $\Phi=0.13$ and $8400 \pm 2820 L_{\odot}$ for $\Phi=0.26$. These luminosities are higher than those predicted by all models for the respective phases except for the $\mathrm{E}$ model series, for which a higher luminosity of the parent star was adopted (see Table 3).

\subsection{CLVs and visibility shapes}

As discussed in Sect. 3.2, there is good agreement between the derived Rosseland linear radii and the Rosseland linear radii predicted by model series $\mathrm{P}$ and $\mathrm{M}$ series models, while there is little or no agreement for the other models. On the other hand, the effective temperatures derived from observations are close to those predicted by the D-model series. In this section we investigate whether one of the model series can be favored by comparing the observed and theoretical visibility shapes.

The measured visibilities at six different phases within the first and second cycle of the observations are shown 


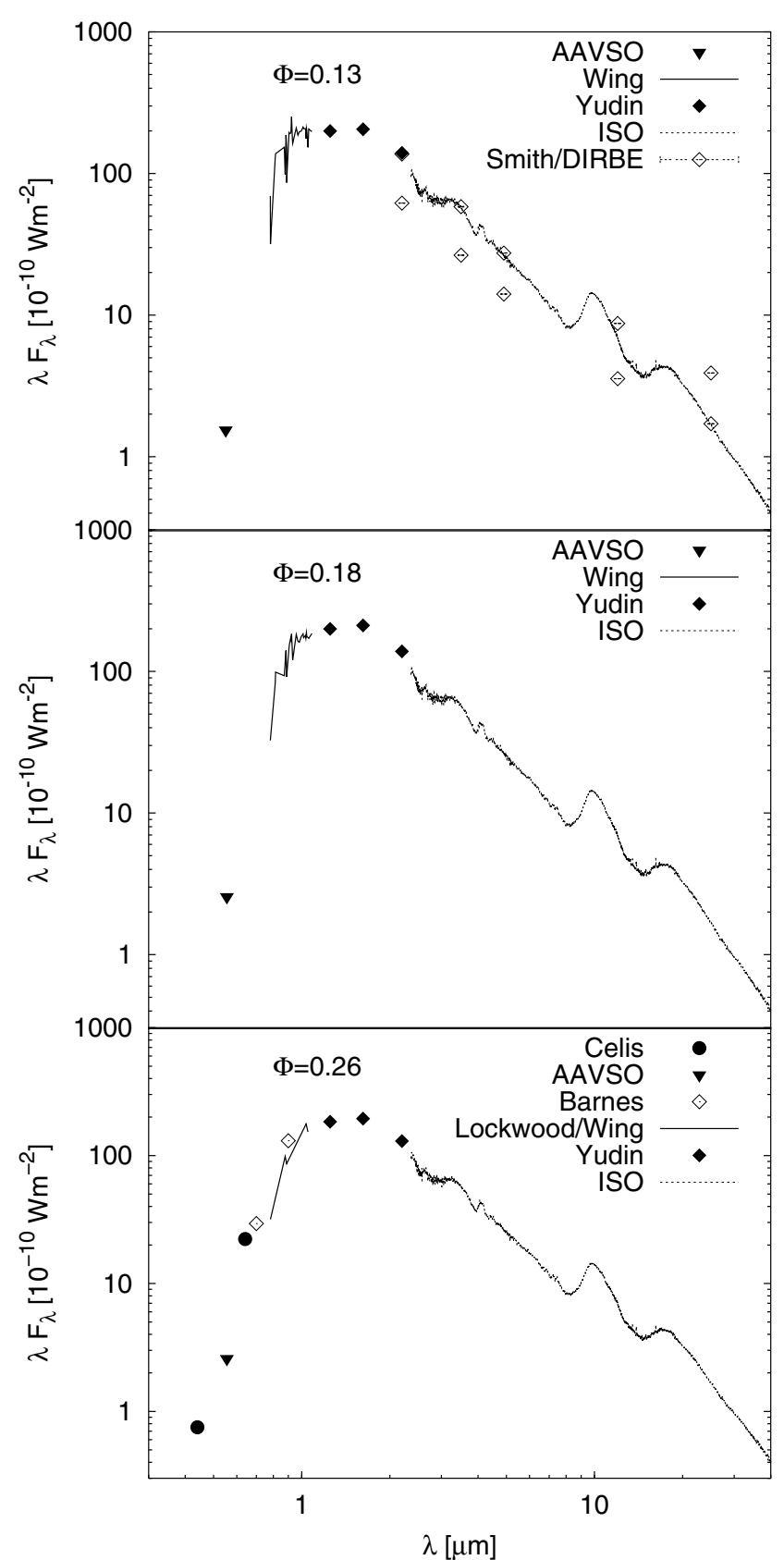

Fig. 5. Spectral energy distributions (SEDs) of $o$ Cet for phase $\Phi=$ 0.13 (top), $\Phi=0.18$ (middle), and $\Phi=0.26$ (bottom). The panels show visual flux densities from Mattei (2003) at the corresponding phase and cycle (triangles), together with the ISO spectrum (longward of $2.36 \mu \mathrm{m}$, dotted line) taken at maximum light. Also included are $J H K$ photometry measurements from Yudin (2003) (filled diamonds) taken at the phase and cycle which correspond to the epoch of the VINCI observations. The top and middle panels show spectrophotometry from Wing (2003) at 26 wavelengths between $0.78 \mu \mathrm{m}$ and $1.10 \mu \mathrm{m}$ (solid line) for the respective phases, and the lower panel shows photometry data at 7 wavelengths: data at $0.44 \mu \mathrm{m}$ and $0.70 \mu \mathrm{m}$ are from Celis (1982) (filled circles), and data between $0.78 \mu \mathrm{m}$ to $1.05 \mu \mathrm{m}$ are from Lockwood \& Wing (1971) (solid line). Further measurements at $0.70 \mu \mathrm{m}$ and $0.90 \mu \mathrm{m}$ for $\Phi=0.23$ were taken from Barnes (1973) (open diamonds). In the top panel we also plotted DIRBE flux densities taken at maximum and minimum light (open diamonds) (Smith et al. 2002) in the 2.2, 3.5, 4.9, 12, and $25 \mu \mathrm{m}$ photometric bandpasses.
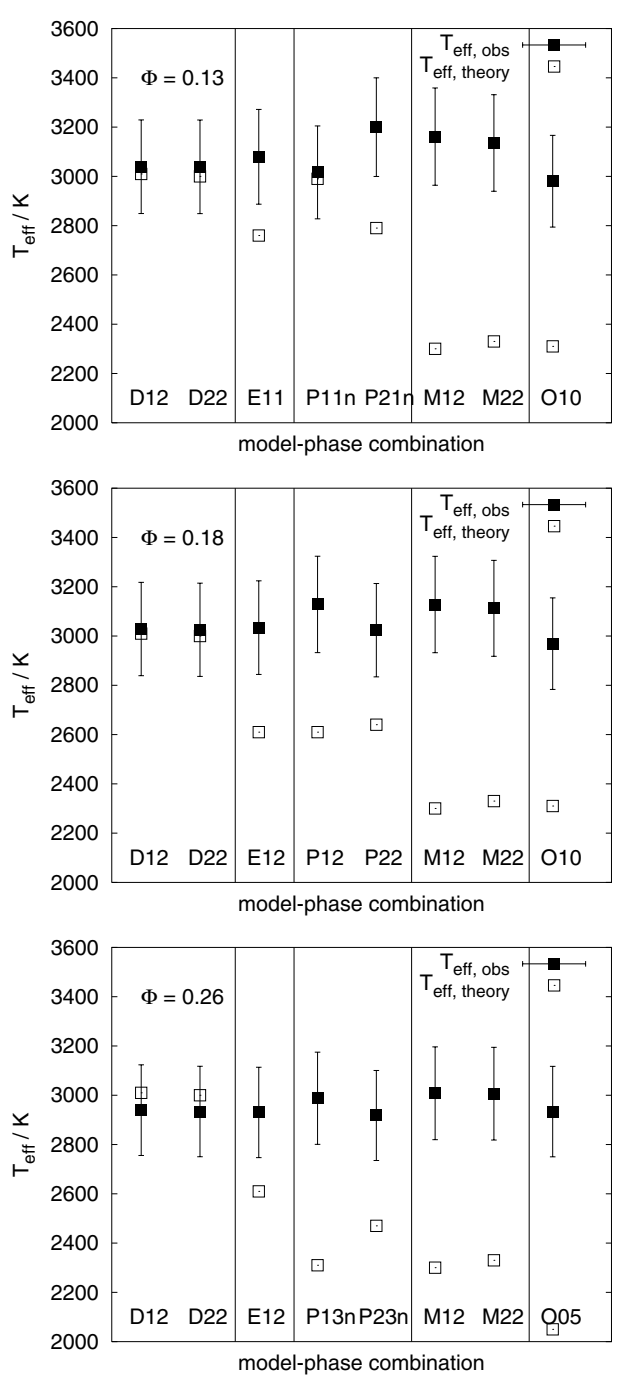

Fig. 6. Comparison of the effective temperatures derived from the observations using Eq. (2) at $\Phi=0.13,0.18$, and 0.26 (filled squares) and the model effective temperatures (open squares). The measured effective temperatures were derived from the measured Rosseland angular radii (see Sect. 3.2) and from our reconstructed SEDs.

in Fig. 2, together with fitted model visibility curves for the P, $\mathrm{M}$, and D models. The P-series fundamental pulsator mode model P21n shows much better agreement with the observation at $\Phi=0.13$ than the $\mathrm{D}$ and $\mathrm{M}$ models or simple UD model CLVs. This can be clearly seen from the inset in Fig. 2, and a comparison of the reduced $\chi^{2}$ values for the different model fits. For model P21n we derived $\chi_{\mathrm{P} 21 \mathrm{n}}^{2}=6.7$, while we obtained $\chi_{\mathrm{D} 22}^{2}=41.5$ and $\chi_{\mathrm{M} 22}^{2}=28.8$ for the models D 22 and M 22, respectively. For the simple uniform-disk model, which is also shown in Fig. 2, we found $\chi_{\mathrm{UD}}^{2}=56.2$. For all other phases shown in Fig. 2, the small number of visibility points and the insufficient coverage of the spatial frequency domain make it impossible to draw conclusions about which model series should be favored. It should be noted that the agreement of the observed CLV shape with the P-series model CLV shape is also better than for the $\mathrm{E}$ and $\mathrm{O}$ firstovertone model series (reduced $\chi^{2}$ values of 22.9 and 45.4, 
respectively), which were already ruled out from radius and temperature comparisons.

The visibility can also be affected by a circumstellar dust shell which is not included in the model calculations. A dust shell could cause a narrow visibility peak at low spatial frequencies. Therefore, we measured the visibilities of $o$ Cet using our speckle camera at the SAO $6 \mathrm{~m}$ telescope. These additional visibilities cover the low spatial frequency domain, showing that the use of dust-free CLV models is justified (see Fig. 2, middle left).

Although the measured visibility shape for $\Phi=0.13$ is in good agreement with the predictions made by the P21n model, we nevertheless wish to point out that the observed visibility shape can also be affected by deviations from circular symmetry of the intensity distribution. Therefore, we studied possible effects of such asymmetries on the visibility shapes by assuming elliptical uniform-disk intensity distributions with ratios of minor to major axis between 0.7 and 1.0. It turned out that the observed visibilities cannot be reproduced by such elliptical uniform disk intensity distributions. However, we note that this study cannot exclude the presence of other types of asymmetries in Mira, such as surface inhomogeneities produced by giant convective cells as suggested by Schwarzschild (1975).

\subsection{Diameter-phase relationship}

Taking the Rosseland diameters derived with the P model CLVs at the four different phases within the first cycle, we obtained the diameter-phase relationship presented in Fig. 7. We do not consider the data within the second cycle of the observations, since the visibilities at $\Phi=1.40$ and $\Phi=1.47$ lie in a completely different spatial frequency range (or baseline range, respectively) compared to the data for $\Phi<1$, and the derived diameters are very sensitive to small differences between the assumed model CLV shape and the actual CLV shape of the object. Figure 7 clearly shows a monotonic increase of the Rosseland angular diameter with decreasing brightness from phase $\Phi=0.13\left(d_{\text {Ross }}^{\mathrm{a}}=28.9 \pm 0.3\right.$ mas, corresponding to a Rosseland linear radius of $R_{\text {Ross }}=332 \pm 38 R_{\odot}$ ) to phase $\Phi=0.40\left(d_{\text {Ross }}^{\mathrm{a}}=34.9 \pm 0.4\right.$ mas, $\left.R_{\text {Ross }}=402 \pm 46 R_{\odot}\right)$. Therefore, as the visual intensity decreases, the $K$-band diameter of $o$ Cet increases. From our analysis we find an $18 \%$ increase of the diameters of $o$ Cet between $\Phi=0.13$ and $\Phi=$ 0.40 . Thompson et al. (2002) found a similar diameter increase for the oxygen-rich Mira star S Lac. They obtained an increase of the angular size of $22 \%$ between maximum $(\Phi=0.0)$ and minimum light $(\Phi=0.5)$. This corresponds to a variation of approximately $14 \%$ between $\Phi=0.13$ and $\Phi=0.40$ (linear interpolation), which is close to our result for $o$ Cet.

Our results of the diameter-phase variation are summarized in Table 5. For comparison, UD angular diameters were also included. As can be seen, $d_{\text {Ross }}^{\mathrm{a}}$ is similar to $d_{\mathrm{UD}}^{\mathrm{a}}$, but the P models agree much better with the measured visibility shapes than the simple UD models, as we discussed in the previous section.

The intensity profiles of the best fitting models for the four different phases, which were found to be the P21n, P22, P23n, and P24n series models, are shown in Fig. 8. The P models are
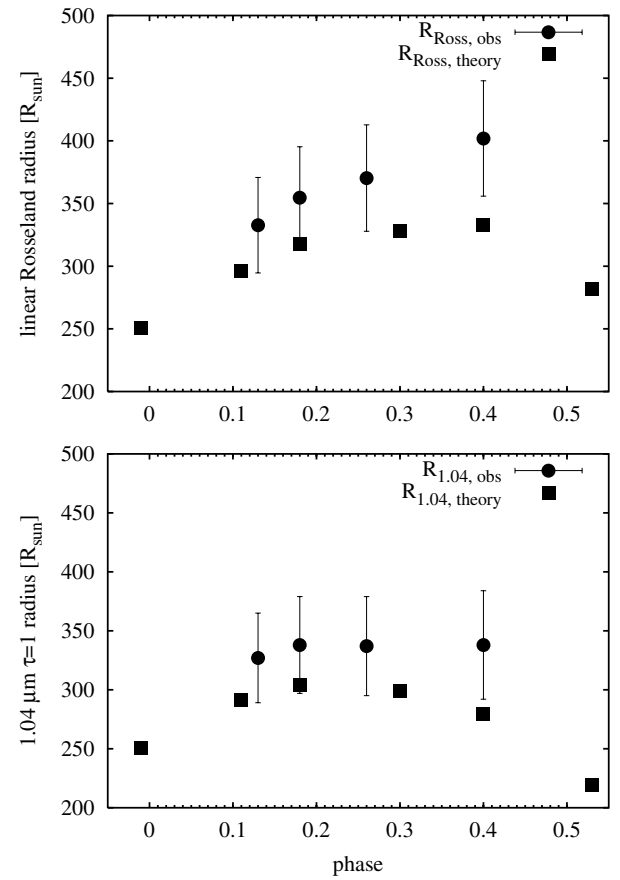

Fig. 7. Top: linear Rosseland radii of $o$ Cet derived from P21n, P22, $\mathrm{P} 23 \mathrm{n}$, and P24n series models (filled circles) at four different phases $\left(\Phi=0.13: R_{\text {Ross }}=332 \pm 38 R_{\odot} ; \Phi=0.18: R_{\text {Ross }}=354 \pm 41 R_{\odot} ;\right.$ $\left.\Phi=0.26: R_{\text {Ross }}=370 \pm 42 R_{\odot} ; \Phi=0.40: R_{\text {Ross }}=402 \pm 46 R_{\odot}\right)$ and theoretical P2 $x$ models (filled squares). The diagram clearly shows a monotonic increase of radius with decreasing brightness, in line with the predictions from TLSW. Bottom: derived (filled circles) and theoretical (filled squares) $R_{1.04}$ radii of $o$ Cet.

among those with the lowest effective temperatures and exhibit extended faint wings near maximum light $(\Phi=0.0)$ and protrusions near minimum light $(\Phi=0.5)$. The extended wing in the intensity profile at maximum light is mainly caused by water molecules, which are one of the most abundant molecules in the atmospheres of oxygen-rich Miras (Tej et al. 2003a). From the analysis of the ISO spectrum, Yamamura et al. (1999) concluded that $\mathrm{H}_{2} \mathrm{O}$ layers are more extended at maximum light. This is in agreement with the CLV near maximum light (top left panel in Fig. 8). As the visual brightness decreases from maximum to minimum phase, the effective temperature decreases, and the extended wings are less pronounced. From $\Phi=0.18$ to $\Phi=0.40$, water absorption increases strongly behind the shock front. This results (i) in a protrusion of the CLV shape as seen in R Leo by Perrin et al. (1999) ("Gaussian-type" CLV) and (ii) in substantial molecular-band blanketing of continuum windows and an increase of $R_{\text {Ross }}^{\mathrm{a}}$, whose position is marked by arrows in Fig. 8 (see the discussion in Tej et al. 2003a). Note that the theoretical $R_{\text {Ross }}^{\mathrm{a}}$, as well as the theoretical $R_{1.04}^{\mathrm{a}}$ that approximately describes "continuum pulsation" (Table 4), reaches their maximum values at $\Phi \sim 0.4$ and $\Phi \sim 0.2$, respectively (Fig. 7), where the difference is caused by the increasing blanketing of near-continuum windows towards the minimum phase. Due to the lack of observations close to minimum and maximum light phases and the size of the error bars of the radii, there is not enough evidence from the VINCI measurements to confirm this behavior of $R_{\text {Ross }}^{\mathrm{a}}$ and $R_{1.04}^{\mathrm{a}}$. Note in 
Table 5. Diameter-phase relationship: the columns give (from left to right): phase $\Phi$, models used for fitting, Rosseland angular diameter $d_{\text {Ross }}^{\text {a }}$ (derived by fitting P model visibility curves from TLSW and ISW to the observed visibilities), Rosseland linear radius $R_{\text {Ross }}$ derived from the observations, theoretical P model Rosseland radius $R_{\text {Ross,model }}$, and UD angular diameter.

\begin{tabular}{cccccc}
\hline \hline$\Phi$ & Model & $d_{\text {Ross }}^{\mathrm{a}}[\mathrm{mas}]$ & $R_{\text {Ross }}\left[R_{\odot}\right]$ & $R_{\text {Ross,model }}\left[R_{\odot}\right]$ & $d_{\mathrm{UD}}^{\mathrm{a}}[\mathrm{mas}]$ \\
\hline 0.13 & P21n & $28.90 \pm 0.29$ & $332 \pm 38$ & 296 & $29.24 \pm 0.30$ \\
0.18 & P22 & $30.82 \pm 0.31$ & $355 \pm 41$ & 318 & $29.53 \pm 0.30$ \\
0.26 & P23n & $32.18 \pm 0.32$ & $370 \pm 42$ & 328 & $30.49 \pm 0.30$ \\
0.40 & P24n & $34.92 \pm 0.35$ & $402 \pm 46$ & 333 & $33.27 \pm 0.33$ \\
\hline
\end{tabular}
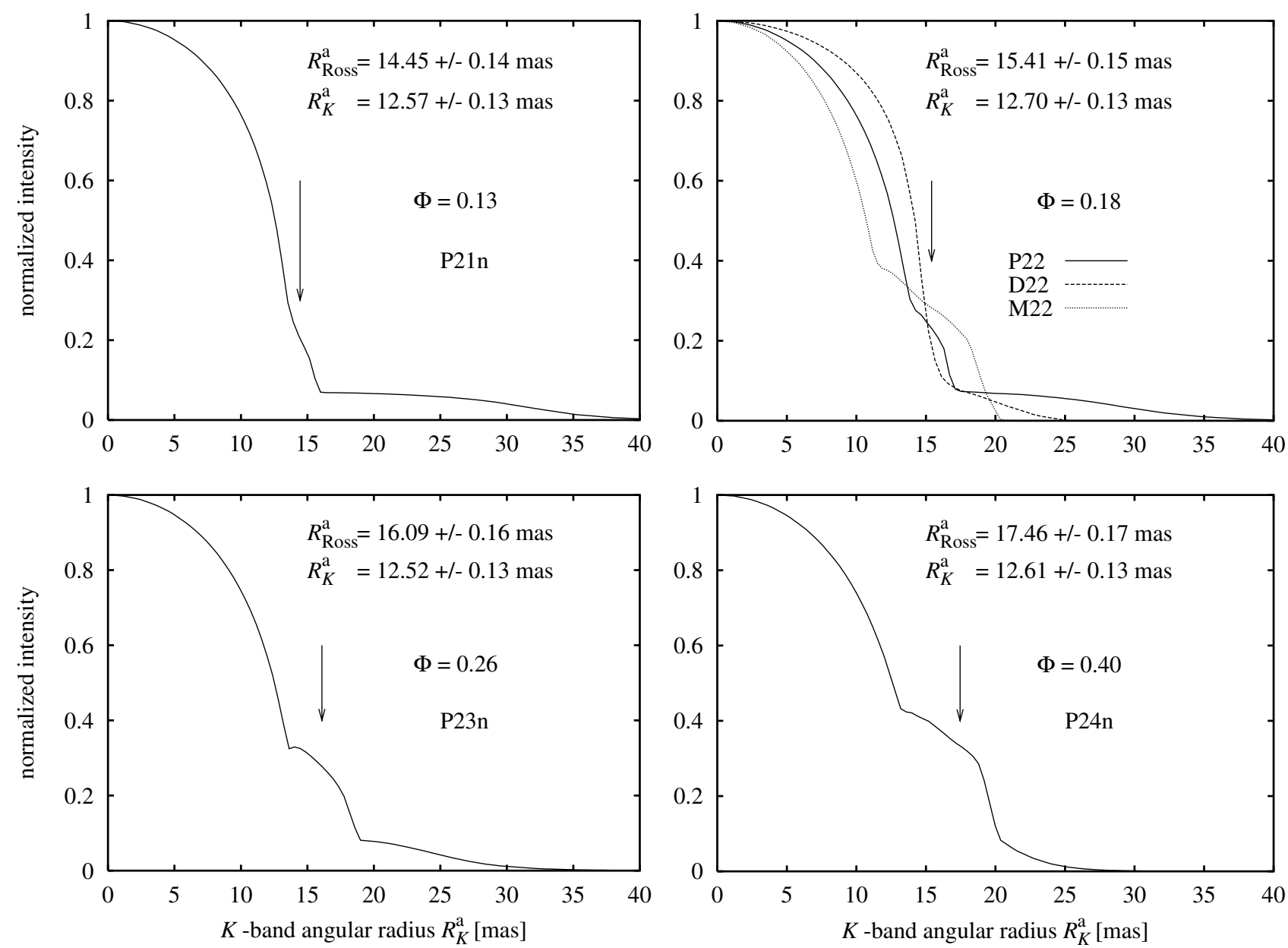

Fig. 8. Theoretical CLV shapes of the ISW model P21n plotted for the $K$-band angular radius $R_{K}^{a}$ corresponding to $o$ Cet at $\Phi=0.13$ (top left), of the TLSW model P22 at $\Phi=0.18$ (top right), of the ISW model P23n at $\Phi=0.26$ (bottom left), and of the ISW model P24n at $\Phi=0.4$ (bottom right) as a function of angular radius. The intensity is normalized to 1 at the disk center. For comparison, the intensity profiles of BSW model D22 (top right, dashed line) and the HSW model M 22 (top right, dotted line) at $\Phi=0.18$ are also shown. The extended wing of the CLV for P models near maximum light is caused by water molecules in the outer region of the atmosphere. The corresponding visibility profiles are shown in Fig. 2. For each phase, the values of the $K$-band angular radii $\left(R_{K}^{\mathrm{a}}\right)$ and the Rosseland angular radii $\left(R_{\text {Ross }}^{\mathrm{a}}\right)$ are given. The arrows mark the position of the Rosseland angular radius. The protrusion seen for phases $\Phi \geq 0.18$ results from the strong increase of water absorption behind a shock front caused by the pulsation of the stellar atmosphere.

this context that the absolute zero-phase point is uncertain by about 0.05 to 0.1 due to the irregularities of both the observed (e.g., Whitelock et al. 2000) and the model-predicted (HSW, ISW) light curves.

The CLVs predicted by the $\mathrm{M}$ models have comparable protrusions, and although the $\mathrm{M}$ model visibility fits to the observed visibilities do not show good agreement, they yield Rosseland linear radii which agree with the theoretical Rosseland linear radii. Because of higher temperatures and lower densities, the D models do not exhibit the protrusions seen in the $\mathrm{P}$ and $\mathrm{M}$ models. Therefore, the slope of the visibility is not as flat as in the case of $\mathrm{P}$ and $\mathrm{M}$ models. For a more detailed description of the different model CLVs, we refer to BSW, HSW, TLSW, and Tej et al. (2003a). 


\section{Conclusions}

We presented $K$-band observations of $o$ Cet obtained with the VLTI and its beam combiner instrument VINCI. From these VINCI observations at six different epochs we derived Rosseland angular radii using 5 different theoretical model series from BSW, HSW, TLSW, and ISW. Using the derived Rosseland angular diameter and the SEDs reconstructed from various photometry and spectrophotometry data, we obtained effective temperatures ranging from $T_{\text {eff }}=3192 \pm 200 \mathrm{~K}$ at $\Phi=0.13$ to $2918 \pm 183 \mathrm{~K}$ for $\Phi=0.26$.

We found that there is fair agreement between the Rosseland linear radii derived from the observed visibilities and those predicted by the fundamental mode pulsation model series $\mathrm{P}$ and $\mathrm{M}$, while there is no agreement for other models. The nonlinear pulsation models all start from a static "parent" star. The parent star for the best-fitting model series, the fundamental-mode $\mathrm{P}$ series, has a radius of $\sim 240 R_{\odot}$. It is clear from Fig. 4 that $o$ Cet, and indeed the nonlinear pulsation models from the $\mathrm{P}$ series, nearly all have radii of $\sim 300-400 R_{\odot}$. Thus, the effect of large amplitude pulsation is to expand the surface layers of the star so that its apparent radius is considerably larger at most phases than the radius it would have if static (note that this effect is milder with respect to a continuum radius like $R_{1.04}$; cf. Table 4 and the discussion in ISW). This expansion of the apparent radius does not greatly affect the interior of the model or the pulsation period. Hence, if one compares observed radii of Miras in (say) a radius-period diagram with the radii and pulsation periods of the parent stars, then one will clearly not find agreement. This is the reason that the Miras were thought for so long to be first-overtone pulsators (see, e.g., Feast 1999; Tuchman 1999, and references therein). It is only by a detailed comparison of interferometric angular diameter measurements with models of large-amplitude, pulsating atmospheres that this problem has been solved.

On the other hand, the effective temperatures derived from the observations are very close to the effective temperatures predicted by the D-model series but higher than those predicted by the $\mathrm{P}$ and $\mathrm{M}$ models. Given the definition of effective temperature $L=4 \pi \sigma R^{2} T_{\text {eff }}^{4}$, this tells us that the P-series models are too low in luminosity, consistent with the luminosities derived in Sect. 3.3 for $o$ Cet (compared to the model values in Table 4). The shape of the measured visibilities for $o$ Cet at phase $\Phi=0.13$ are best fitted with the P model series, whereas all other model series and simple UD models show much poorer agreement with the observations. Taking all this into account, it is clear that a higher-luminosity, fundamentalmode model series is required for a more accurate modeling of $o$ Cet.

Furthermore, we found that the observed visibility functions and diameters change considerably from phase 0.13 to phase 0.40. The Rosseland angular diameter of $o$ Cet increases from $d_{\text {Ross }}^{\text {a }}=28.9 \pm 0.3$ mas (corresponding to a Rosseland linear radius of $R_{\text {Ross }}=332 \pm 38 R_{\odot}$ ) at $\Phi=0.13$ to $d_{\text {Ross }}^{\text {a }}=$ $34.9 \pm 0.4$ mas $\left(R_{\text {Ross }}=402 \pm 46 R_{\odot}\right)$ at $\Phi=0.4$. Thus, the diameter of $o$ Cet increases by $18 \%$ between these two phases, which is in good agreement with the approximately $14 \%$ diameter increase derived from linear interpolation of the results by Thompson et al. (2002) for the oxygen-rich Mira S Lac.

Acknowledgements. We acknowledge with thanks the variable star observations from the AAVSO International Database contributed by observers worldwide and used in this research. This research was in part supported (M. Scholz, P. R. Wood) by the Australian Research Council and the Deutsche Forschungsgemeinschaft within the linkage project "Red Giants". We would like to thank Prof. Robert Wing for providing his spectrophotometry measurements. In addition, we would like to thank Dr. Boris Yudin for his valuable JHK photometry data. These interferometric measurements have been obtained using the Very Large Telescope Interferometer, operated by the European Southern Observatory at Cerro Paranal, Chile. We thank the VLTI Team (Garching and Paranal) for its support in this research. All the VINCI data presented in this paper are public and were retrieved from the ESO/ST-ECF Archive (Garching, Germany). Finally, we thank the referee, Dr. D. Mourard, for his many useful comments which helped to improve the manuscript.

\section{References}

Böhm-Vitense, E. 1958, Z. Astrophys., 46, 108

Barnes, T. G. 1973, ApJS, 25, 369

Baschek, B., Scholz, M., \& Wehrse, R. 1991, A\&A, 246, 374

Bessell, M. S., Brett, J. M., Wood, P. R., \& Scholz, M. 1989, A\&A, 213, 209

Bessell, M. S., Scholz, M., \& Wood, P. R. 1996, A\&A, 307, 481

Bonneau, D., Foy, R., Blazit, A., \& Labeyrie, A. 1982, A\&A, 106, 235

Bonneau, D., \& Labeyrie, A. 1973, ApJ, 181, L1

Burns, D., Baldwin, J. E., Boysen, R. C., et al. 1998, MNRAS, 297, 462

Celis, S. L. 1982, AJ, 87, 1791

Coudé du Foresto, V., Ridgway, S., \& Mariotti, J.-M. 1997, A\&AS, 121,379

Coudé du Foresto, V., \& Ridgway, S. T. 1992, in High-Resolution Imaging by Interferometry II, ed. F. Merkle, \& J. M. Boeckers, ESO Proc., 731

Danchi, W. C., Bester, M., Degiacomi, C. G., Greenhill, L. J., \& Townes, C. H. 1994, AJ, 107, 1469

ESA. 1997, VizieR Online Data Catalog, 1239, 1

Feast, M. 1999, in Asymptotic Giant Branch Stars, ed. T. Le Bertre, A. Lèbre, \& C. Waelkens, IAU Symp., 191, 109

Feast, M. W. 1996, MNRAS, 278, 11

Haniff, C. A., Ghez, A. M., Gorham, P. W., et al. 1992, AJ, 103, 1662

Haniff, C. A., Scholz, M., \& Tuthill, P. G. 1995, MNRAS, 276, 640

Hofmann, K.-H., Scholz, M., \& Wood, P. R. 1998, A\&A, 339, 846

Hofmann, K.-H., Balega, Y., Scholz, M., \& Weigelt, G. 2000a, A\&A, 353,1016

Hofmann, K.-H., Beckmann, U., Blöcker, T., et al. 2000b, in Interferometry in Optical Astronomy, ed. P. J. Léna, \& A. Quirrenbach, SPIE Proc., 4006, 688

Hofmann, K.-H., Balega, Y., Scholz, M., \& Weigelt, G. 2001, A\&A, 376,518

Ireland, M. J., Scholz, M., \& Wood, P. R. 2004, MNRAS, in press

Jacob, A. P., \& Scholz, M. 2002, MNRAS, 336, 1377

Karovska, M., Nisenson, P., Papaliolios, C., \& Boyle, R. P. 1991, ApJ, 374, L51

Kervella, P., Gitton, P. B., Segransan, D., et al. 2003, in Interferometry for Optical Astronomy II, ed. W. A. Traub, SPIE Proc., 4838, 858

Knapp, G. R., Pourbaix, D., Platais, I., \& Jorissen, A. 2003, A\&A, 403, 993

Labeyrie, A. 1970, A\&A, 6, 85 
Labeyrie, A., Koechlin, L., Bonneau, D., Blazit, A., \& Foy, R. 1977, ApJ, 218, L75

Lockwood, G. W., \& Wing, R. F. 1971, ApJ, 169, 63

Mariotti, J.-M., Coudé du Foresto, V., Perrin, G., Zhao, P., \& Léna, P. 1996, A\&AS, 116, 381

Mattei, J. A. 2003, private communication

Meisner, J. A. 2003, Ap\&SS, 286, 119

Pease, F. G. 1931, Ergebn. d. Exakten Naturwiss., 10, 84

Perrin, G. 2003, A\&A, 400, 1173

Perrin, G., Coudé du Foresto, V., Ridgway, S. T., et al. 1999, A\&A, 345, 221

Quirrenbach, A., Mozurkewich, D., Armstrong, J. T., et al. 1992, A\&A, 259, L19

Richichi, A., \& Percheron, I. 2002, A\&A, 386, 492

Robertson, B. S. C., \& Feast, M. W. 1981, MNRAS, 196, 111

Scholz, M. 1985, A\&A, 145, 251

Scholz, M. 2003, in Interferometry for Optical Astronomy II, ed. W. A. Traub, SPIE Proc., 4838, 163

Scholz, M., \& Takeda, Y. 1987, A\&A, 186, 200

Schwarzschild, M. 1975, ApJ, 195, 137

Smith, B. J. 2003, AJ, 126, 935

Smith, B. J., Leisawitz, D., Castelaz, M. W., \& Luttermoser, D. 2002, AJ, 123, 948

Tej, A., Lançon, A., \& Scholz, M. 2003a, A\&A, 401, 347

Tej, A., Lançon, A., Scholz, M., \& Wood, P. R. 2003b, A\&A, 412, 481

Thompson, R. R., Creech-Eakman, M. J., \& van Belle, G. T. 2002, ApJ, 577, 447
Tuchman, Y. 1999, in Asymptotic Giant Branch Stars, ed. T. Le Bertre, A. Lèbre, \& C. Waelkens, IAU Symp., 191, 123

Tuthill, P. G., Haniff, C. A., \& Baldwin, J. E. 1994, in Very High Angular Resolution Imaging, ed. J. G. Robertson, \& J. T. William, IAU Symp., 158, 395

van Belle, G. T., Dyck, H. M., Benson, J. A., \& Lacasse, M. G. 1996, AJ, 112, 2147

Watanabe, T., \& Kodaira, K. 1979, PASJ, 31, 61

Weigelt, G., Balega, Y., Hofmann, K.-H., \& Scholz, M. 1996, A\&A, 316, L21

Weigelt, G., Mourard, D., Abe, L., et al. 2000, in Interferometry in Optical Astronomy, ed. P. J. Léna, \& A. Quirrenbach, SPIE Proc., 4006, 617

Weigelt, G., Beckmann, U., Berger, J., et al. 2003, in Interferometry for Optical Astronomy II, ed. W. A. Traub, SPIE Proc., 4838, 181

Weiner, J., Danchi, W. C., Hale, D. D. S., et al. 2000, ApJ, 544, 1097

Whitelock, P., Marang, F., \& Feast, M. 2000, MNRAS, 319, 728

Wilson, R. W., Baldwin, J. E., Buscher, D. F., \& Warner, P. J. 1992, MNRAS, 257, 369

Wing, R. 2003, private communication

Wittkowski, M., Aufdenberg, J. P., \& Kervella, P. 2004, A\&A, 413, 711

Wood, P. R., Alcock, C., Allsman, R. A., et al. 1999, in Asymptotic Giant Branch Stars, ed. T. Le Bertre, A. Lèbre, \& C. Waelkens, IAU Symp., 191, 151

Yamamura, I., de Jong, T., \& Cami, J. 1999, A\&A, 348, L55

Yudin, B. 2003, private communication 